First Peoples Child \& Family Review

A Journal on Innovation and Best Practices in Aboriginal Child Welfare Administration,

Research, Policy \& Practice

\title{
Les mauvais traitements envers les enfants autochtones signalés à la Protection de la jeunesse du Québec: Comparaison Interculturelle
}

\author{
Marc Tourigny, Pascale Domond, Nico Trocmé, Bruno Sioui and Karine Baril
}

Volume 3, Number 3, 2007

URI: https://id.erudit.org/iderudit/1069399ar

DOI: https://doi.org/10.7202/1069399ar

See table of contents

Publisher(s)

First Nations Child and Family Caring Society of Canada

ISSN

1708-489X (print)

2293-6610 (digital)

Explore this journal

Cite this article

Tourigny, M., Domond, P., Trocmé, N., Sioui, B. \& Baril, K. (2007). Les mauvais traitements envers les enfants autochtones signalés à la Protection de la jeunesse du Québec: Comparaison Interculturelle. First Peoples Child \& Family Review, 3(3), 84-102. https://doi.org/10.7202/1069399ar

\section{Article abstract}

L'étude vise à décrire les signalements impliquant des enfants autochtones et à les comparer à ceux impliquant des enfants non-autochtones à partir d'un échantillon représentatif des signalements faits aux services québécois de protection de la jeunesse. Les résultats démontrent qu’au Québec, les situations impliquant des enfants autochtones, par rapport à celles impliquant des enfants non-autochtones, se caractérisent par l'occupation d'un logement subventionné, l'abus de substances chez les parents, un nombre plus élevé d'enfants dans la famille, le fait que le signalement soit reçu en urgence sociale et le fait que le signalement provienne moins souvent de la mère. Les enjeux pour l'intervention auprès des familles autochtones et des recommandations pour les recherches futures sont discutés.
Copyright @ $@$ Marc Tourigny, Pascale Domond, Nico Trocmé, Bruno Sioui, Karine Baril, 2007
This document is protected by copyright law. Use of the services of Erudit (including reproduction) is subject to its terms and conditions, which can be viewed online.

https://apropos.erudit.org/en/users/policy-on-use/ 


\title{
Les mauvais traitements envers les enfants autochtones signalés à la Protection de la jeunesse du Québec: Comparaison Interculturelle*
}

\author{
Marc Tourigny $^{a}$, Pascale Domond ${ }^{b}$, Nico Trocméc, Bruno Sioui ${ }^{a}$, et Karine Barila
}

\author{
'Département de psychoéducation de l'Université de Sherbrooke. \\ 'Département de psychologie de l'UQAM \\ ‘École de travail social de l’Université McGill
}

\section{Introduction}

La question des mauvais traitements envers les enfants dans les communautés autochtones ${ }^{1}$ soulève depuis plusieurs années de nombreux débats et fait l'objet de préoccupations importantes de la part des services de protection de l'enfance canadiens et québécois. Pour l'ensemble du Canada (hormis le Québec), Blackstock, Trocmé et Bennett (2004) ont comparé des données relatives aux mauvais traitements envers les enfants autochtones à celles se rapportant aux enfants non-autochtones d'origine canadienne et non-autochtones d'autres origines ethniques. Les résultats des analyses comparatives effectuées ont permis de connaître l'incidence du phénomène des mauvais traitements pour deux groupes et ont révélé des différences significatives quant aux types de mauvais traitements, au contexte dans lequel ils émergent ainsi qu'aux conséquences qui en découlent. Ces différences laissent croire en l'importance de tenir compte des différences interculturelles dans l'étude des mauvais traitements envers les enfants au Canada.

Remerciements: Les auteurs souhaitent remercier les intervenants de la direction de la protection de la jeunesse pour leur précieuse participation à ce projet. Les demandes de copies devraient être adressées à Marc Tourigny, Département de psychoéducation, Faculté d'éducation, Université de Sherbrooke, 2500, boul. de l'Université, Sherbrooke (Québec), Canada, J1K2R1. (e-mail: Marc.Tourigny@USherbrooke.ca)

\section{Résumé}

L'étude vise à décrire les signalements impliquant des enfants autochtones et à les comparer à ceux impliquant des enfants non-autochtones à partir d'un échantillon représentatif des signalements faits aux services québécois de protection de la jeunesse. Les résultats démontrent qu'au Québec, les situations impliquant des enfants autochtones, par rapport à celles impliquant des enfants non-autochtones, se caractérisent par l'occupation d'un logement subventionné, l'abus de substances chez les parents, un nombre plus élevé d'enfants dans la famille, le fait que le signalement soit reçu en urgence sociale et le fait que le signalement provienne moins souvent de la mère. Les enjeux pour l'intervention auprès des familles autochtones et des recommandations pour les recherches futures sont discutés.

Cette approche comparative a des retombées positivestantauplandel'avancementdesconnaissances que sur le plan de la prévention et du traitement, car elle permet de contribuer au développement d'organisation de services et de programmes d'intervention adaptés aux besoins spécifiques des familles autochtones et non-autochtones touchées par ce problème. Cependant, aucune information n'est disponible à ce jour concernant les différences interculturelles pouvant exister concernant les mauvais traitements chez les enfants autochtones et non-autochtones au Québec. Le but de cette étude est de palier à ce manque d'information en examinant les différences et similarités pouvant exister entre les dossiers des enfants autochtones et non autochtones signalés aux services de protection de la jeunesse du Québec.

\section{Population autochtone du Québec}

Selon la Loi sur les Indiens, « un Indien est une personne qui est inscrite dans le Registre des Indiens du 
Ministère des Affaires Indiennes et du Nord Canadien (MAINC) ou qui a le droit de l'être ", distinguant ainsi les Indiens inscrits, les Indiens non inscrits et les Inuits (Secrétariat aux affaires autochtones, 2001). La population autochtone québécoise n'est pas homogène puisqu'elle est composée de dix nations amérindiennes et de la nation inuite, réparties dans près d'une soixantaine de communautés sur tout le territoire québécois et dont $70 \%$ occupe les terres réservées (Secrétariat aux affaires autochtones, 2001). En 2001, l'Enquête auprès des peuples autochtones (EAPA) a démontré que le Québec comptait une population autochtone de 79400 personnes, soit $8 \%$ de tous les Autochtones du Canada et 1\% de l'ensemble de la population du Québec (Statistique Canada, 2003). De ce nombre, 68735 étaient, en 2005, des Indiens inscrits et 46709 demeuraient sur une réserve (MAINC, 2006). Selon cette même enquête, 34090 enfants vivaient durant cette période dans une famille autochtone, représentant environ $2 \%$ des enfants québécois. Soixante huit pourcent (68\%) d'entre eux avaient entre 0 et 14 ans, un pourcentage supérieur à ce que l'on observait pour les enfants nonautochtones pour ce même groupe d'âge (59\%).

De 1996 à 2001, la population autochtone s'est accrue plus rapidement que l'ensemble de la population du Québec, ayant augmenté de 11\%, comparativement à $1 \%$ pour l'ensemble de la population québécoise. Les rapports plus récents de 2005 montrent que la population autochtone du Québec s'est accrue depuis l'année 2001 (Secrétariat aux affaires autochtones, 2005). Composée de 82 824 personnes, cette augmentation laisse croire que le nombre d'enfants autochtones ait également considérablement augmenté depuis les informations disponibles en 2001.

\section{Historique des populations autochtones du Canada: politiques de colonisation et d'assimilation}

Bon nombre de rapports historiques portant sur les populations autochtones du Canada convergent sur le fait qu'au cours des 500 ans ayant suivi l'arrivée des Européens, et plus particulièrement au cours des années ayant suivi l'adoption de la Loi sur les Indiens en 1876, qui a fait en sorte que tous les Indiens sont devenus sous la tutelle du gouvernement canadien, un bouleversement social a été engendré chez les Autochtones, perturbant ainsi leurs valeurs et leur mode de vie traditionnels. Dans certains cas, des communautés autochtones auraient été complètement anéanties par les politiques et les mesures assimilatrices canadiennes ${ }^{2}$ (Commission royale sur les peuples autochtones, 1996; Dupuis, 2001). En effet, leurs enfants auraient été enlevés ; leur gouvernement, leur économie et leurs traditions règlementés ou bannis par des lois (Armitage, 1995; Bennett \& Blackstock, 2002; Fournier \& Crey, 1997; Réame \& Macklem, 1994). Le refus du droit de vote, la règlementation de l'identité, l'interdiction d'acheter des terres, la proscription des cérémonies spirituelles, la relocalisation vers des réserves et la ségrégation imposée dans celles-ci, la restriction sur le plan des droits civils et politiques, l'expropriation des terres en vertu de lois (p.ex., la Loi sur les Indiens) et l'obligation pour des enfants autochtones de fréquenter des pensionnats où il appert qu'une forte proportion d'Autochtones, aujourd'hui âgés de plus de 35 ans, auraient été abusés physiquement et agressés sexuellement (Commission de la santé et des services sociaux des Premières Nations du Québec et du Labrador, 2006) sont des exemples de politiques racistes et oppressives à l'égard des peuples autochtones rapportés dans ces rapports.

\section{Premiers systèmes de protection et de bien- être de l'enfance appliqués aux populations autochtones}

Dès le début des années 1870, le gouvernement fédéral commença à établir des écoles résidentielles pour enfants autochtones âgés de 5 à 16 ans dans le but d'enseigner à la jeunesse autochtone comment devenir des membres productifs de la société selon les lignes de conduite européennes et chrétiennes (Miller, 1996). Les enfants étaient donc retirés de leurs foyers et de leurs communautés pour être placés dans ces pensionnats dirigés conjointement par le gouvernement et différentes communautés religieuses. L'assimilation des Autochtones s'effectuait alors par l'endoctrinement des enfants afin qu'ils abandonnent leur style de vie et leur langue. Ces écoles existaient en vertu de la Loi sur les Indiens de 1876 et se sont répandues dans toutes les provinces, particulièrement celles de l'ouest canadien et à l'exception des provinces maritimes (Miller, 1996). L'enfance autochtone aurait également été la cible d'exploitation et de maltraitance par le remplacement, dans les années 1960 , des pensionnats au profit des premiers systèmes 
de protection et de bien-être de l'enfance comme système de soins pour les enfants autochtones du Canada (Armitage, 1995). Toutefois, l'implantation de cette nouvelle organisation ne répondait toujours pas aux besoins des Autochtones et renforçait plutôt la désintégration des familles de ces communautés (Bennett \& Blackstock, 2002). À titre d'exemple illustrant l'inadéquation de la politique des systèmes de bien-être et de protection de l'enfance visant les Autochtones, les conditions de vie et la pauvreté, plutôt que la maltraitance, auraient été les raisons principales pour lesquelles de nombreux enfants autochtones ont été retirés de leurs familles. Même si les enfants étaient placés en raison de situations d'abus ou de négligences graves, les conditions de vie ou les soins médicaux étaient souvent la raison de l'intervention des systèmes de protection (Tiechroeb, 1997).

À la fin des années 1960 au Canada, environ 30\% à $40 \%$ des enfants suivis par les systèmes de protection de l'enfance auraient été des enfants autochtones, alors qu'ils représentaient moins de $4 \%$ de la population canadienne (Fournier \& Crey, 1997). Dès 1983³, la surreprésentation des enfants autochtones dans les systèmes de protection de l'enfance était étendue à l'ensemble du pays, avec notamment des taux variant entre $50 \%$ et $70 \%$ d'enfants autochtones en tutelle pour les provinces des prairies (Armitage, 1995; Fournier \& Crey, 1997; Tiechroeb, 1997).

Selon Tiechroeb (1997), une faible proportion de ces enfants retournait dans leur famille d'origine après avoir été placés dans des familles d'accueil ou adoptés. Ceux qui retournaient à la maison après des absences prolongées se retrouvaient aliénés de leur famille et de leur environnement culturel (Hudson \& McKenzie, 1985). En effet, " élevés par des parents [non-autochtones] de classe moyenne, ils ont grandi avec une connaissance et une compréhension limitées de leurs racines. " (Bennett \& Blackstock, 2002, p.21). De plus, l'agression sexuelle et l'abus physique de ces enfants de la part des parents d'accueil ou des parents adoptifs auraient été rapportés par certains d'entre eux (Tiechroeb, 1997).

\section{Les répercussions des politiques sociales sur les peuples autochtones}

La relation entre le gouvernement du Canada et les peuples des Premières Nations, marquée par la pression sociale, économique, politique et culturelle résultant des politiques assimilatrices, a eu des conséquences dévastatrices auprès des communautés autochtones du pays. Ces politiques de colonisation, d'expropriation et d'assimilation ont entraîné des répercussions notamment sur leurs systèmes familiaux, par le bris des liens familiaux, et leur situation économique (Bennett \& Blackstock, 2002; Fournier \& Crey, 1997; Hudson, 1987; Kimelman, 1985). Selon Bennett et Blackstock (2002), l'application forcée des services provinciaux de bien-être et de protection de l'enfance chez les familles autochtones n'a fait qu'exacerber les effets dévastateurs subis par ces peuples au cours de la colonisation et qui perdurent encore à ce jour, notamment en termes de problèmes psychosociaux et socioéconomiques. Parmi ces problèmes sociaux, signalons la pauvreté, la violence conjugale, les mauvais traitements envers les enfants, la criminalité et l'alcoolisme.

\section{La pauvreté}

Les données du recensement de 1991 analysées dans le rapport du Conseil Canadien de Développement Social montrent que les Autochtones vivant en milieu urbain au Canada ont deux fois plus de risques de vivre dans la pauvreté que les non-Autochtones ${ }^{4}$ (Lochhead \& Shillington, 1996). En effet, alors que le taux de pauvreté moyen pour tous les habitants en milieu urbain était de $25 \%$, le taux de pauvreté des personnes autochtones vivant en milieu urbain s'élevait à $56 \%$ durant cette période. Dans le même sens, le taux de pauvreté des mères canadiennes chefs de famille monoparentale s'élevait à $45 \%$, alors qu'il atteignait $73 \%$ chez les mères autochtones (Conseil national du bien-être social, 2000). De plus, en 1995, 60\% des enfants autochtones de moins de six ans vivaient au sein d'une famille pauvre, comparativement à $25 \%$ pour l'ensemble des enfants canadiens (Conseil national du bien-être social, 2000). Les conditions de vie observées dans les milieux autochtones ont également un impact sur l'espérance de vie moyenne de ces enfants, qui est de huit ans inférieure à la moyenne nationale une fois qu'ils atteignent l'âge adulte (Fournier \& Crey, 1997). Les données plus récentes montrent que le problème de pauvreté chez les enfants autochtones est encore d'actualité, puisque selon le recensement canadien de 2001, 40\% des enfants autochtones vivant à l'extérieur des réserves sont pauvres (Statistique Canada, 2003). 
Pour ce qui est du Québec, les données liées aux profils de la population autochtone du Québec du recensement de 2001 nous indiquent que la pauvreté frappe les familles autochtones de cette province. À titre d'exemple, on constate que la moitié de la population autochtone âgée de 15 ans et plus vivant au Québec, soit 25880 personnes, avait un revenu inférieur à $15330 \$$ durant cette période. Peu de données québécoises récentes nous permettent de connaître le taux de pauvreté chez les Autochtones comparativement aux non-Autochtones, mais les données présentées en 1999 par le Conseil Canadien de Développement Social révélaient que 37\% des Autochtones vivaient en 1995 sous le seuil de faible revenu établi par Statistique Canada, comparativement à 23\% des Québécois (Schetagne, 1999).

\section{Éducation, emploi et chômage}

La situation d'emploi et d'éducation chez les jeunes Autochtones apparaît plus difficile que chez les non-Autochtones. En effet, 14\% des Amérindiens, $17 \%$ des Métis et 19\% des Inuits vivant hors réserve étaient chômeurs, comparativement à $8 \%$ des nonAutochtones (Statistique Canada, 2003). Ainsi, dans l'ensemble, les Autochtones de 15 ans et plus affichent des taux de chômage beaucoup plus élevés que les non-Autochtones. En ce qui concerne le niveau d'éducation, alors que 19\% de tous les Canadiens de 25 ans et plus auraient obtenu un diplôme universitaire (Bowlby, 2002), c'est le cas pour seulement 8\% des Autochtones au Canada dans la même tranche d'âge (Kunz, Milan \& Schetagne, 2001; Statistique Canada, 2003).

Selon Statistique Canada (2003), au Québec, concernant le taux de diplomation universitaire, on estimait en 2001 chez les personnes âgées de 25 ans et plus à seulement à $6 \%$ la proportion des Autochtones ayant obtenu un grade universitaire au niveau du baccalauréat ou à un niveau supérieur (contre $10 \%$ de la population totale); et à $48 \%$ la proportion ayant un niveau inférieur au diplôme d'études secondaires (comparativement à 39\% pour la population totale).

Enmatière d'emploi, les Autochtones représentaient en $20012 \%$ de la population active au Québec. Toutefois, le taux de chômage dans cette population se situait à 18\% durant cette période (MAINC, 2006).

À notre connaissance, nous ne disposons pas de données permettant de croire que le problème de faible scolarisation et de chômage soit largement résolu pour les Autochtones du Québec. Bien qu'il $\mathrm{y}$ ait eu une nette amélioration de la scolarisation des jeunes Autochtones au cours des trois dernières décennies, le faible taux de scolarisation des populations autochtones rapporté en 2001 démontre que le problème affecte plus que la moitié des jeunes autochtones adolescents et adultes.

\section{Violence conjugale}

Les femmes autochtones sont davantage à risque que les autres Canadiennes d'être victimes de violence conjugale. L'enquête sociale générale sur la violence conjugale menée au Québec en 1999 révèle que $25 \%$ des femmes autochtones auraient été agressées par un conjoint ou un ex-conjoint au cours des cinq dernières années, comparativement à $8 \%$ des femmes non-autochtones (Jiwani, 2000). De plus, la probabilité d'être tuées par leur conjoint lors d'une rupture d'union serait huit fois plus élevée chez les femmes autochtones que chez les non-autochtones. Dans le même sens, les formes les plus graves de violence, soit celles qui pourraient mettre la vie en péril (être battue, étranglée, menacée avec une arme à feu ou un couteau ou agressée sexuellement) seraient également plus courantes chez les femmes victimes autochtones.

\section{Abus de substances et criminalité}

La consommation d'alcool serait un problème grave au sein des communautés autochtones. En effet, Bennett et Blackstock (2002, p.40) citent ce que la Commission royale sur les peuples autochtones (1996) a conclu à cet effet, soit que « l'alcool est la substance la plus intoxicante qui risque d'entraîner le plus grand nombre de problèmes chez les peuples et les communautés autochtones au Canada.». Une étude ontarienne utilisant des données de 1985-86 a quantifié la consommation d'alcool par comté, et elle a comparé les comtés avec réserves autochtones aux comtés sans réserves (Adrian, Payne, \& Williams, 1991). Les comtés avec réserves avaient un pourcentage supérieur de consommation d'alcool comparativement à celui du reste des comtés et la présence des réserves expliquait $25 \%$ de variation de la consommation d'alcool dans la province. L'étude a permis d'établir un lien direct entre le niveau faible de revenu et la consommation d'alcool, où chaque 
$1000 \$$ supplémentaire ajouté aux bénéfices après impôts était mis en corrélation avec une diminution de 0,3 litre de la consommation totale d'alcool.

On ne dispose pas de données spécifiques pour le Québec concernant la criminalité au sein de la communautéautochtone, maislesdonnées canadiennes témoignent de la présence de ce problème social. À cet effet, les résultats de l'enquête sociale générale sur la violence conjugale révèlent qu'environ $35 \%$ de la population autochtone canadienne avait déclaré avoir été victime d'au moins un crime dans les 12 mois précédant l'enquête, comparativement à $26 \%$ chez les non-Autochtones (Jiwani, 2000).

Les jeunes Autochtones, comparativement aux non-Autochtones, sont présents en plus grande proportion dans tous les aspects du système de justice pénale au Canada. Bien qu'ils ne constituaient que 5\% de la population en 2001-2002, ils comptaient pour près de $25 \%$ des placements en détention provisoire, $22 \%$ des placements en garde ordonnée, $17 \%$ des mises sous probation et $16 \%$ des applications de mesures de rechange. Ces taux ne seraient toutefois pas uniformes d'une province à l'autre (Gouvernement du Canada, 2005).

\section{Signalements pour mauvais traitements dans la communauté autochtone}

Les rapports annuels des ministères provinciaux et territoriaux des services de protection de l'enfance estimaient, pour les années 2000-2002, que 7600 enfants étaient en placement au Canada et $80 \%$ de ces enfants seraient des Autochtones (Farris-Manning \& Zandrastra, 2003). Quant à l'évolution du nombre d'Autochtones impliqués dans les services de protection de l'enfance, le MAINC (2006) révèle que les cas de signalement pour protection de l'enfance dans la communauté autochtone ont augmenté de $70 \%$ de 1995 à 2003.

\section{Liens entre les problèmes sociaux et les mau- vais traitements envers les enfants}

Plusieurs études montrent que les mauvais traitementsenverslesenfants, notammentlanégligence, sont fortement associés au contexte socioéconomique dans lequel l'enfant évolue, caractérisé notamment par un faible niveau de scolarisation de la mère, des ressources socioéconomiques peu élevées, le chômage chez les parents, l'abus de substances des parents et la violence conjugale(Black, Heyman, \& Slep, 2001; Gelles \& Hargreaves, 1990; O’Keefe, 1995; Schumacher, Slep, \& Heyman, 2001; Sedlak, 1997; Zuravin, 1987).

Malgré le lien existant entre les difficultés socioéconomiques des familles et l'occurrence des mauvais traitements chez les enfants, peu de données à ce sujet sont disponibles pour les communautés autochtones. En effet, aucune donnée québécoise ne semble exister concernant les liens entre les mauvais traitements chez les enfants autochtones et les facteurs de risque sociaux-économiques et concernant la spécificité culturelle des situations de mauvais traitements signalées à la protection de la jeunesse et impliquant un enfant autochtone.

\section{Comparaison des mauvais traitements dans des familles autochtones et non-autochtones}

Deux études canadiennes, comparant les mauvais traitements dans la communauté autochtone et nonautochtone, ont été répertoriées. Dans une étude exploratoire, Leung et Carter (1983) ont examiné les différences culturelles concernant les mauvais traitements envers des enfants d'origine chinoise, autochtone ainsi que canadienne-anglophone ayant un dossier à l'hôpital général de Vancouver. Bien que cette étude présente plusieurs limites méthodologiques, elle suggère que dans les familles autochtones, le syndrome d'alcoolisme fœtal, la négligence (sous forme de «failure to thrive») et les ecchymoses seraient plus présentes en comparaison aux familles canadiennes anglaises. Quant à l'identification de l'auteur des mauvais traitements, les résultats ont démontré qu'il $\mathrm{y}$ a une prépondérance des mères comme auteurs des mauvais traitements pour les cas autochtones, alors que pour les cas canadiens-anglophones, on identifie une proportion plus importante d'agresseurs qui n'ont pas de lien de parenté avec l'enfant (conjoint de la mère, gardien d'enfants et autres).

Blackstock et al. (2004) ont réalisé à partir d'une vaste enquête canadienne une série d'analyses comparatives entre les signalements d'enfants autochtones, ceux d'enfants non-autochtones d'origine canadienne (hormis ceux d'origine québécoise) et ceux des non-autochtones d'autres origines ethniques faits aux services canadiens de protection de la jeunesse ${ }^{5}$. 
Les résultats ont démontré que la situation des enfants autochtones se caractérise, comparativement aux situations des deux autres groupes, par une plus grande proportion de familles: 1) vivant de l'aide sociale; 2) vivant dans un logement insalubre; 3 ) ayant reçu des services de la protection de l'enfance dans le passé; 4) impliquées dans des situations de négligence; et 5) de familles ayant déménagé dans les 6 derniers mois.

En ce qui concerne les parents autochtones, comparativement au deux autres groupes, on retrouve des différences significatives quant: 1) à la plus grande proportion d'entre eux à avoir vécu des mauvais traitements dans leur enfance; 2) aux problèmes d'abus de substances (drogue/alcool) qui sont davantage présents; 3) à la plus forte présence d'activités criminelles; 4) à l'isolement social plus présent; 5) à une plus grande présence de maladie mentale ou de problèmes de fonctionnement cognitifs; et 6) à l'âge moins élevé des parents. Enfin, toujours selon cette étude, le fait d'être un enfant autochtone augmenterait les probabilités que les mauvais traitements soient jugés fondés et que l'enfant soit placé au moment de la prise en charge.

Chez les enfants, comparés aux deux autres groupes, les enfants autochtones se différencient principalement par une plus forte présence de problèmes d'abus de substances et d'absentéisme scolaire.

\section{Limites méthodologiques}

Malgré l'avancement des connaissances apportées par ce type d'études comparatives, des limites méthodologiques se doivent d'être soulevées. Une de ces limites concerne le fait qu'il est possible que des différences entre les Autochtones et les nonAutochtones soient davantage attribuables au niveau économique plus faible des familles autochtones et non à des différences d'ordre culturel. Comme le niveau économique est une variable fortement associée aux mauvais traitements envers les enfants, et comme les familles autochtones sont nettement plus défavorisées économiquement, le contrôle du niveau économique permettrait de mieux dégager les différences liées à la culture. Une seconde limite a trait à l'utilisation d'analyses univariées pour déterminer les différences entre les groupes. Cette stratégie ne permet pas de dégager l'apport unique de chacune des variables permettant de caractériser chacun des groupes par rapport à l'autre. Des stratégies d'analyses multivariées semblent plus intéressantes à cet égard.
La présente étude vise donc à examiner le phénomène des mauvais traitements d'enfants autochtones signalés aux services de protection de la jeunesse du Québec à partir d'un échantillon représentatif des signalements québécois. Plus spécifiquement, deux objectifs ont été fixés, le premier visant à décrire les signalements impliquant des enfants autochtones et le second à comparer ces signalements à des signalements impliquant des enfants non-autochtones. Afin de palier à certaines limites méthodologiques identifiées précédemment, les deux groupes d'enfants ont été appareillés quant au revenu familial, à la région administrative où vit la famille et aux figures parentales vivant dans le milieu de vie de l'enfant.

\section{Méthodologie}

\section{Procédure}

Laprésenteétudeportesurdes analyses secondaires réalisées à partir d'une banque de données provenant d'une enquête (étude d'incidence québécoise - ÉIQ ${ }^{6}$ ) qui visait à documenter tous les signalements reçus et retenus entre le 1er octobre et le 31 décembre 1998. Les signalements proviennent de 16 des 18 régions administratives du Québec, chacune desservie par une Direction de la protection de la jeunesse (DPJ). Deux régions ont été exclues de l'enquête en raison de leur faible nombre par rapport à l'ensemble de la population québécoise et des difficultés d'accessibilité résultant de leur éloignement : le Nunavik, dont la population est de 8000 habitants et les Terres Cries de la Baie James, dont la population est de 9000 habitants.

Le taux de signalements retenus et documentés (taux de réponse) au cours de l'étude a été calculé en divisant le nombre de signalements documentés par le nombre de signalements enregistrés par les DPJ au cours de la même période (Tourigny et al., 2002). Pour l'ensemble des signalements retenus $(\mathrm{N}=4929)$, le taux moyen de questionnaires complétés est de $86 \%$ (variant de $64 \%$ à $99 \%$ selon les DPJ).

L'échantillon final de notre étude a été tiré des signalements retenus. Un signalement est défini comme "Toute situation d'un enfant de 0-17 ans, rapportée au directeur de la protection de la jeunesse par une personne qui pense que la sécurité ou le développement de cet enfant est ou peut être compromis " (Ministère de la Santé et des Services Sociaux, 1988: 5). 


\section{Échantillon}

Un premier échantillon a été constitué de 215 enfants dont le signalement a été retenu et qui vivaient avec au moins un parent autochtone. Pour chaque enfant de cet échantillon, un enfant nonautochtone comparable a été sélectionné par la suite. L'appariement des deux groupes s'est fait à partir des caractéristiques suivantes : 1) le signalement provient du même Centre Jeunesse ; 2) les figures parentales présentes dans le milieu de vie de l'enfant; et 3) le revenu familial annuel. Il a été impossible de réaliser un appariement individuel parfait, car pour un certain nombre d'enfants autochtones, il nous a été impossible de trouver un enfant non-autochtone présentant exactement les mêmes caractéristiques. Toutefois, des analyses de Khi-carré montrent que les deux groupes ne se distinguent pas significativement sur ces trois variables. L'échantillon final comprend donc 215 paires d'enfants. Le Tableau 1 montre que les enfants des deux groupes vivaient principalement avec leurs deux parents biologiques $(42 \%)$ et près du tiers des enfants vivaient dans une famille monoparentale (31\%). Les enfants vivaient dans des familles ayant un très faible revenu, puisque plus de la moitié (56\%) vivait dans une famille dont le revenu était inférieur à $15000 \$(40 \%)$ ou provenait de l'aide sociale (16\%), le quart $(22 \%)$ vivait dans une famille dont le revenu familial se situait entre 15000 et $25000 \$$. Malgré de légères différences entre les deux groupes concernant cette variable, il n'y avait toutefois aucune différence statistique significative. Enfin, au niveau de la région, $30 \%$ des enfants provenaient de la Côte-Nord, 15\% de l'Abitibi-Témiscaminque, $17 \%$ de la région de Montréal et 37\% provenaient du reste du Québec.

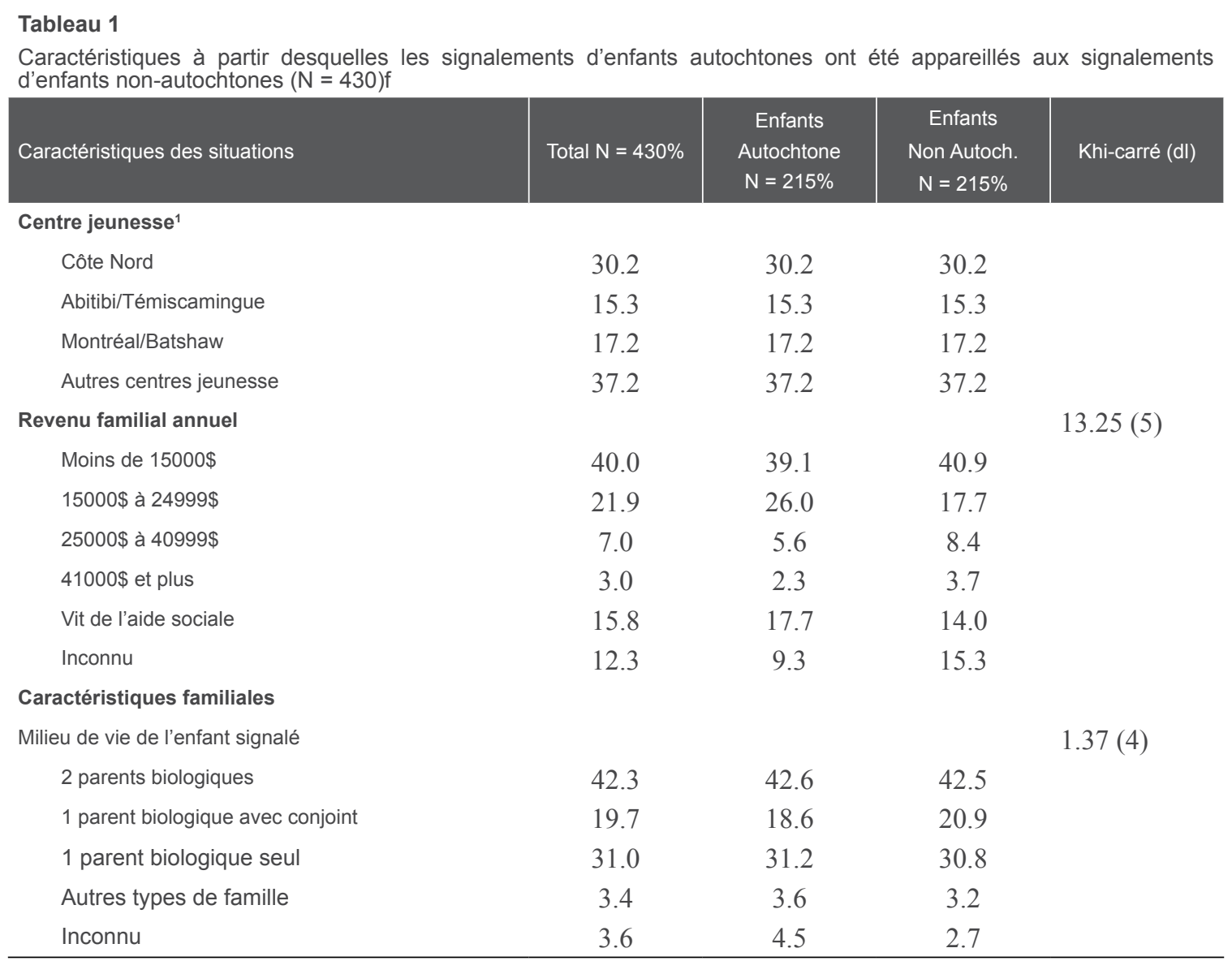

(1) Aucun test statistique n'a été effectué pour cette variable puisque l'appariement était parfait. 


\begin{tabular}{|c|c|c|}
\hline Variables & $\begin{array}{l}\text { Canada } \\
\text { (Trocmé) }\end{array}$ & $\begin{array}{l}\text { Quebec } \\
\text { (Tourigny) }\end{array}$ \\
\hline $\begin{array}{l}\text { Structure familiale* } \\
2 \text { parents biologiques } \\
\text { Recomposée } \\
\text { Monoparentale }\end{array}$ & $\begin{array}{l}21.9 \\
21.6 \\
56.5\end{array}$ & $\begin{array}{l}42.1 \\
18.6 \\
31.2\end{array}$ \\
\hline $\begin{array}{l}\text { Source de revenus } \\
\text { temps plein } \\
\text { temps partiel } \\
\text { aide sociale } \\
\text { Autre }\end{array}$ & $\begin{array}{l}15.8 \\
10.2 \\
58.1 \\
16.0\end{array}$ & $\begin{array}{c}12.4 \\
7.2 \\
74.6 \\
5.7\end{array}$ \\
\hline Logement non sécuritaire & 7.9 & 10.5 \\
\hline $\begin{array}{l}\text { Déménagement } \mathbf{1 2} \text { derniers mois* } \\
\text { Aucun } \\
1 \\
2 \text { ou plus }\end{array}$ & $\begin{array}{l}60.1 \\
22.9 \\
17.0\end{array}$ & $\begin{array}{l}68.8 \\
18.3 \\
12.9\end{array}$ \\
\hline $\begin{array}{l}\text { Caractéristiques des mauvais } \\
\text { traitements* } \\
\text { Non fondé } \\
\text { Fondé } \\
\text { Suspecté } \\
\text { Abus physique } \\
\text { Abus sexuel } \\
\text { Négligence } \\
\text { Abus psychologique } \\
\text { Abandon } \\
\text { Troubles comportement } \\
\text { Violence conjugale }\end{array}$ & $\begin{array}{c}26.3 \\
49.5 \\
24.2 \\
18.6 \\
10.1 \\
57.9 \\
6.5 \\
\mathrm{Nd} \\
\mathrm{Nd} \\
7.0 \\
\end{array}$ & $\begin{array}{c}13.5 \\
80.9 \\
5.6 \\
14.9 \\
11.6 \\
62.3 \\
26.0 \\
7.4 \\
19.5 \\
\mathrm{Nd}\end{array}$ \\
\hline $\begin{array}{l}\text { Caractéristiques de l'enfant } \\
\text { Abus de subst. naissance } \\
\text { Anxiété ou dépression } \\
\text { Nombre de problèmes } 0 \\
\text { Nombre de problèmes } 1 \\
\text { Nombre de problèmes 2+ }\end{array}$ & $\begin{array}{c}6.1 \\
7.6 \\
77 \\
12.7 \\
10.3\end{array}$ & $\begin{array}{c}2.5 \\
7.4 \\
80.2 \\
11.9 \\
7.9\end{array}$ \\
\hline $\begin{array}{l}\text { Caractéristiques des parents } \\
\text { Age: } \\
30 \\
31-40 \\
41-50 \\
51+ \\
\text { Histoire de maltraitance* } \\
\text { Abus alcool + drogues* } \\
\text { Activités criminelles } \\
\text { Santé mentale } \\
\text { Santé physique } \\
\text { Faible soutien social }\end{array}$ & $\begin{array}{c}49.5 \\
44.7 \\
5.4 \\
0.4 \\
47.2 \\
91.0 \\
17.2 \\
21.7 \\
7.0 \\
33.9\end{array}$ & $\begin{array}{c}39.7 \\
43.1 \\
13.9 \\
3.3 \\
86.9 \\
53.2 \\
8.9 \\
15.8 \\
9.8 \\
29.4\end{array}$ \\
\hline
\end{tabular}

\section{Variables}

Le formulaire d'enquête pour documenter les signalements retenus a été élaboré principalement à partir d'instruments de mesures provenant d'enquêtes similaires dont 1'Ontario Incidence Study (Trocmé, McPhee, Tam, \& Hay, 1994), le National Incidence Study - NIS (Sedlak \& Broadhurst, 1996) et l'Étude Canadienne d'Incidence - ÉCI (Trocmé et al., 2001). Le formulaire a été complété à la fin de l'étape Orientation par l'intervenant de la protection de la jeunesse responsable de cette étape. Il a ainsi permis de recueillir de l'information sur les caractéristiques du signalement, des mauvais traitements fondés, de l'enfant signalé, des adultes jouant le rôle de parents auprès de l'enfant, du milieu de vie de l'enfant signalé et des services durant l'évaluation du signalement.

\section{Caractéristiques du signalement retenu}

Des questions portaient sur la description de la situation signalée, telles que la source de signalement (10 sources de signalements distinctes), le type de réception du signalement (service régulier et urgence sociale), et le fait que l'enfant ait fait l'objet d'un signalement retenu dans les 12 mois précédant l'étude ou d'une prise en charge au cours des cinq années précédant l'étude.

\section{Problématiques fondées}

La présence de sept problématiques (abus physique, abus sexuel, négligence, abandon, mauvais traitements psychologiques, troubles de comportement sérieux et autres) jugées fondées suite à l'évaluation faite par l'intervenant de la protection de la jeunesse était documentée. Les définitions des problématiquesprovenaientdel'inventaireconcernant le bien-être de l'enfant en relation avec l'exercice des responsabilités parentales - ICBE (Vézina \& Bradet, 1990), du NIS (Sedlack, 1991), de l'ÉCI (Trocmé et al., 2001) et du Système clientèle jeunesse - SCJ (Trudeau \& Pellan, 1998) pour la problématique des troubles de comportement sérieux.

\section{Caractéristiques de l'enfant, de sa famille et des figures parentales}

Le formulaire d'enquête a permis d'obtenir de l'information sur les caractéristiques démographiques de l'enfant (âge et sexe) et sur la présence de huit problèmes vécus par l'enfant (problèmes à la naissance, retard du développement, handicap de l'ouïe, de la vue ou de la parole, problème chronique de santé, problème d'apprentissage, hyperactivité, problèmes psychiatriques, dépression/anxiété).

Concernant les variables familiales, la sécurité ou non du logement, le fait que la famille soit propriétaire du logement ou non, le nombre d'enfants signalés dans la famille, la présence d'une autre personne significative pour l'enfant (autre que les figures parentales), le 
nombre de déménagements dans les 12 derniers mois et le nombre d'enfants dans la famille ont été mesurées.

Dix problèmes, connus ou soupçonnés, (violence conjugale, consommation d'alcool ou de drogue, criminalité, santé mentale, santé physique, faible soutien social, retard mental, problèmes financiers séparation/divorce ou autre) permettaient de décrire les difficultés vécues par les figures parentales. Pour la présente étude, nous avons utilisé le nombre total de problèmes vécus par la figure parentale principale et les problèmes qui se sont avérés statistiquement significatifs entre les deux groupes. Le niveau de coopération du parent a été évalué à partir de l'échelle de l'ICBE, une échelle en quatre points allant de «adéquat» à «sérieusement inadéquat» (Vézina \& Bradet, 1990) et le questionnaire permettait également de documenter si le parent avait lui-même vécu des mauvais traitements dans son enfance.

\section{Stratégies d'analyse}

Des tests de khi-carré et des tests-t ont d'abord été effectués afin de comparer les enfants autochtones et nonautochtones(version 9.0 deSPSS). Pourlepremierobjectif, ces comparaisons ont porté sur 28 variables à l'étude (Tableau 2). Les 16 variables qui s'avéraient significatives ont été utilisées dans une analyse de régression logistique par blocs hiérarchiques afin d'identifier les variables qui prédisaient l'appartenance aux groupes d'enfants. Lorsque les variables indépendantes sont nombreuses et intercorrélées, l'approche hiérarchique diminue le nombre de variables à se retrouver simultanément dans le modèle, ce qui améliore la performance des procédés d'estimation. Aussi, la comparaison des résultats, d'une étape à l'autre, fournit quelques détails supplémentaires concernant les liens entre les variables indépendantes. L'ordre d'entrée des trois grandes catégories de variables était le suivant, soit : les caractéristiques du signalement, les problématiques fondées et les caractéristiques liées à l'enfant signalé et sa famille.

\section{Résultats \\ Analyses univariées}

Les résultats provenant des analyses de Khi-carré révèlent un certain nombre de différences importantes et significatives entre les dossiers de signalement d'enfants autochtones et ceux d'enfants non-autochtones (voir Tableau 3).
Tableau 2

Variables utilisées pour l'analyse univariée afin de comparer les deux groupes

Caractéristiques du signalement retenu

1. Type de réception*

2. Signalement antérieur retenu (12 mois) NS

3. Prise en charge antérieure ( 5 ans $)^{*}$

4. Sources de signalement - mère*

5. Sources de signalement - famille élargie*

Variables liées à la problématique fondée

6. Présence d'un abus sexuel NS

7. Présence d'un abus physique NS

8. Présence de négligence*

9. Présence d'abandon NS

10. Présence de mauvais traitements psychologiques NS

11 Présence de troubles de comportement*

12. Présence d'une problématique autre NS

Variables liées à l'enfant signalé et sa famille

13. Sexe de l'enfant NS

14. Âge de l'enfant NS

15. Nombre de problèmes connus chez l'enfant*

16. Nombre de besoins de services pour l'enfant NS

17. Milieu de vie de l'enfant NS

18. Nombre d'enfants signalés dans la même famille*

19. Nombre d'enfants dans la famille *

20. Présence d'une autre personne significative NS

21. Nombre de déménagements NS

22. Type d'occupation *

23. Logement non sécuritaire *

24. Niveau de coopération du parent ${ }^{\star}$

25. Mauvais traitements dans l'enfance du parent*

26. Nombre de problèmes connus chez le parent*

27. Présence de violence conjugale vécu par le parent*

28. Présence d'abus de drogue et d'alcool chez le parent*

${ }^{9}$ Neuf autres sources de signalement ont été documentés et testés statistiquement mais aucune différence significative entre les deux n'a été détecté.

\section{Caractéristiques du signalement retenu}

Les résultats portant sur les caractéristiques du signalement retenu démontrent que les signalements impliquant des enfants autochtones étaient plus souvent reçus par les urgences sociales ( $26 \%$ vs $12 \%)$ et que les enfants autochtones avaient plus fréquemment fait l'objet d'une prise en charge par les services de protection de l'enfance aux cours des cinq dernières années, comparativement aux enfants non-autochtones ( $25 \%$ vs $14 \%$ ). Deux différences significatives ont été détectées quant à la proportion des différentes sources de signalements: dans les signalements impliquant des enfants autochtones, on retrouvait moins souvent de signalements faits par la mère de l'enfant signalé (7\% vs $15 \%$ ) et plus souvent un signalement provenant d'un 


\section{First Peoples Child \& Family Review, Volume 3, Number 3, 2007}

\begin{tabular}{|c|c|c|c|c|}
\hline \multicolumn{5}{|c|}{$\begin{array}{l}\text { Tableau } 3 \\
\text { Caractéristiques des signalements, des enfants et leur familles selon que les enfants sont Autochtones ou non }\end{array}$} \\
\hline Caractéristiques des situations & Total $\mathrm{N}=430 \%$ & $\begin{array}{l}\text { Enfants Autochtone } \\
\qquad \mathrm{N}=215 \%\end{array}$ & $\begin{array}{l}\text { Enfants on autoch. } \\
\qquad \mathrm{N}=215 \%\end{array}$ & Khi-carré (dl) \\
\hline \multicolumn{5}{|l|}{ Caractéristiques du signalement retenu } \\
\hline \multicolumn{5}{|l|}{$\begin{array}{ll}\text { Type de réception: } & \text { Services réguliers } \\
& \text { Urgence sociale }\end{array}$} \\
\hline \begin{tabular}{|ll} 
Prise en charge antérieure (5 ans) & Oui \\
& Non
\end{tabular} & $\begin{array}{l}19.2 \\
80.8\end{array}$ & $\begin{array}{l}24.8 \\
75.2\end{array}$ & $\begin{array}{l}13.7 \\
86.3\end{array}$ & $8.35^{* *}(1)$ \\
\hline Source de signalement-mère & $\begin{array}{l}10.9 \\
89.1\end{array}$ & $\begin{array}{l}7.0 \\
93.0\end{array}$ & $\begin{array}{l}14.9 \\
85.1\end{array}$ & $6.90^{* *}(1$ \\
\hline \multirow[t]{2}{*}{\begin{tabular}{|ll} 
Source de signalement-famille élargie & Oui \\
& Non
\end{tabular}} & $\begin{array}{l}80.9 \\
89.1\end{array}$ & $\begin{array}{l}16.0 \\
83.3\end{array}$ & $\begin{array}{l}0.1 \\
9.1 \\
94.9\end{array}$ & $14.92^{\star \star \star}(1)$ \\
\hline & \\
\hline \multicolumn{2}{|l|}{ Présence de négligence $\quad$ Oui } & 48.4 & $\begin{array}{l}36.7 \\
63.3\end{array}$ & $5.95^{*}(1)$ \\
\hline \multirow{2}{*}{\begin{tabular}{|ll} 
Présence de troubles de comportements & $\begin{array}{c}\text { Oui } \\
\text { Non }\end{array}$ \\
Variables liées à l'enfant signalé et sa famille
\end{tabular}} & $\begin{array}{l}2.4 \\
25.3 \\
74.1\end{array}$ & $\begin{array}{l}17.0 \\
82.3\end{array}$ & $\begin{array}{l}0.0 \\
33.0 \\
67.0\end{array}$ & $13.38^{* \star *}(1)$ \\
\hline & \multicolumn{4}{|c|}{ Variables liées à l'enfant signalé et sa famille } \\
\hline \begin{tabular}{|ll} 
Ãge de l'enfant: & $0-5$ ans \\
& $6-11$ ans
\end{tabular} & $\begin{array}{l}38.9 \\
29.5 \\
31.6\end{array}$ & $\begin{array}{l}40.7 \\
31.8 \\
27.5\end{array}$ & $\begin{array}{l}37.1 \\
27.2 \\
35.7\end{array}$ & $4.37(2)$ \\
\hline \multicolumn{5}{|l|}{ Variables liées à l'enfant signalé et sa famille (suite) } \\
\hline $\begin{array}{l}\text { Nombre de problèmes connus chez l'enfant } \\
\text { Aucun } \\
1 \text { problème } \\
2 \text { problèmes } \\
3 \text { problèmes et plus } \\
\end{array}$ & $\begin{array}{l}73.9 \\
16,2 \\
6.5 \\
3.4 \\
\end{array}$ & $\begin{array}{l}80.2 \\
11.9 \\
5.4 \\
2.5\end{array}$ & $\begin{array}{l}67.9 \\
20.3 \\
7.5 \\
4.2 \\
\end{array}$ & $8.28^{*}(3)$ \\
\hline \begin{tabular}{|l}
3 problèmes et plus \\
Nbre d'enfants dans la famille
\end{tabular} & & & & \\
\hline $\begin{array}{l}\text { Nbre d'enfants dans la famille } \\
1 \text { seul enfant }\end{array}$ & 33.7 & 29.8 & 37.7 & $28.18^{* * *}(5)$ \\
\hline 2 enfants & 27.4 & 22.3 & 32.6 & \\
\hline 3 enfants & 17.9 & 16.7 & 19.1 & \\
\hline 4 enfants & 11.6 & 17.2 & 6.0 & \\
\hline \multirow{2}{*}{$\begin{array}{l}5 \text { enfants } \\
6 \text { enfants }\end{array}$} & 5.8 & 8.4 & 3.3 & \\
\hline & 3.5 & 5.6 & 1.4 & \\
\hline Nbre d'enfants signalés dans la famille & & & & \\
\hline 1 seul enfant & 51.4 & 44.7 & 58.1 & $11.96^{*}(4)$ \\
\hline 2 enfants & 21.2 & 22.8 & 19.5 & \\
\hline 3 enfants & 14.9 & 15.3 & 14.4 & \\
\hline 4 enfants & 8.4 & 11.2 & 5.6 & \\
\hline 5 enfants et plus & 4.2 & 6.0 & 2.3 & \\
\hline Type d'occupation du logement & & & & \\
\hline Logement subventionné & 25.9 & 35.7 & 16.6 & $56.67^{* * \star}(3)$ \\
\hline Logement non subventionné & 36.9 & 27.6 & 45.9 & \\
\hline Propriétaire & 26.4 & 17.9 & 34.6 & \\
\hline Autres & 10.7 & 18.9 & 2.9 & \\
\hline Variables liées à l'enfant signalé et sa famille (suite) & & & & \\
\hline $\begin{array}{ll}\text { Logement sécuritaire } & \text { Oui } \\
& \text { Non }\end{array}$ & $\begin{array}{l}92.2 \\
7.8\end{array}$ & $\begin{array}{l}89.5 \\
10.5\end{array}$ & $\begin{array}{c}94.9 \\
5.1\end{array}$ & $4.02^{*}(1)$ \\
\hline Niveau de coopération du parent & & & & \\
\hline Adéquat & 63.7 & 56.7 & 70.7 & $10.60^{*}(3)$ \\
\hline Légèrement inadéquat & 16.0 & 20.5 & 11.6 & \\
\hline Modérément ou sérieusement & 18.6 & 20.5 & 16.7 & \\
\hline \begin{tabular}{|l} 
N'a pas été contacté \\
Mauvais traitements dans
\end{tabular} & $\frac{1.6}{75.3}$ & $\frac{2.3}{86.9}$ & $\frac{0,9}{61.5}$ & $20.66^{* * *}(1)$ \\
\hline l'enfance du parent & 24.7 & 13.1 & 38.5 & \\
\hline Présence de violence conjugale & & & & \\
\hline Non & 68.7 & 62.1 & 75.2 & $11.35^{\star *}(2)$ \\
\hline Connu & 22.2 & 24.8 & 19.6 & \\
\hline Soupçonné & 9.1 & 13.1 & 5.1 & \\
\hline Présence d'abus de drogue/alcool chez le parent & & & & \\
\hline Non & 60.7 & 46.7 & 74.8 & \\
\hline Connu & 27.8 & 43.9 & 11.7 & $55.51^{* * *}(2)$ \\
\hline Soupçonné & 11.4 & 9.3 & 13.6 & \\
\hline Nbre de problèmes connus chez le parent & & & & \\
\hline Aucun & $\begin{array}{l}30,8 \\
28,3\end{array}$ & $\begin{array}{l}26.6 \\
29.4\end{array}$ & 35.0 & $15.47^{* \star}(3)$ \\
\hline $\begin{array}{l}1 \text { problème } \\
2 \text { problèmes }\end{array}$ & $\begin{array}{l}28.3 \\
19.6\end{array}$ & $\begin{array}{l}29.4 \\
19.2\end{array}$ & $\begin{array}{l}27.1 \\
20.1\end{array}$ & \\
\hline $\begin{array}{l}2 \text { problèms } \\
3 \text { problèmes e }\end{array}$ & $\begin{array}{l}19.6 \\
21.2\end{array}$ & $\begin{array}{l}19.2 \\
24.8\end{array}$ & $\begin{array}{l}20.1 \\
17.7\end{array}$ & \\
\hline
\end{tabular}

1. Le ratio de cotes estimé est une mesure de l'augmentation de la cote dans une catégorie par rapport à l'autre, il identifie les catégories les plus à risque.

2. L'intervalle de confiance du ratio de côtes est une mesure de la précision du ratio de côtes estimé.

3. La statistique de Wald identifie les catégories où le phénomène est le plus fréquent. Cependant, si la probabilité est supérieure à 0.05 , on ne peut rejeter l'hypothèse qu'il n'y a pas de différence entre les catégories.

4. La statistique du rapport de vraisemblance est un indicateur de la capacité de chacune des variables à prédire le phénomène, au-delà de la contribution des autres variables incluses dans le modèle. La probabilité tient compte du nombre de paramètres utilisés par la variable pour prédire le phénomène.

(c) Marc Tourigny, Pascale Domond, Nico Trocmé, Bruno Sioui, et Karine Baril 
membre de la famille élargie (17\% vs 5\%).

\section{Problématique fondée}

Quant aux données relatives aux problématiques qui ontétéjugées fondées lors del'évaluation du signalement, on note une proportion plus grande d'enfants victimes de négligence chez les Autochtones (48\% vs $37 \%$ ) et une proportion plus faible d'enfants ayant des troubles du comportement (18\% vs 33\%). La proportion d'enfants vivant des abus sexuels, psychologiques ou physiques est semblable chez les deux groupes.

\section{Caractéristiques des enfants, des parents et des familles signalés}

Les données se rapportant aux caractéristiques des enfants signalés démontrent que les cas autochtones et non-autochtones ne se distinguaient pas en proportion quant à l'âge de l'enfant. Toutefois, les enfants autochtones présentaient moins de problèmes connus comparativement aux enfants non-autochtones. En effet, $80 \%$ des enfants autochtones signalés ne présentaient aucun des problèmes documentés dans l'étude comparativement à $68 \%$ des enfants non-autochtones.
Les familles autochtones comptaient davantage d'enfants comparativement aux non-autochtones. En effet, $48 \%$ des enfants autochtones vivaient dans une famille ayant trois enfants ou plus contre $30 \%$ pour les enfants non-autochtones. De même, 53\% des enfants autochtones vivaient dans une famille où au moins deux enfants avaient été signalés, comparativement à $42 \%$ pour les enfants non-autochtones. Enfin, une plus grande proportion d'enfants autochtones vivait dans un logement subventionné ( $36 \%$ vs $17 \%$ ) et non sécuritaire (11\% vs $5 \%$ ), en comparaison aux enfants non-autochtones.

Concernant les parents, le niveau de coopération des parents autochtones était plus souvent inadéquat que celui des parents non-autochtones (43\% vs $29 \%$ ). Ces premiers ont plus souvent été victimes de mauvais traitements dans leur enfance ( $87 \%$ vs $62 \%)$, impliqués dans des situations de violence conjugale connues ou soupçonnées ( $38 \%$ vs $25 \%$ ) et impliqués dans des problèmes d'abus de substances connus ou soupçonnés ( $53 \%$ vs $25 \%$ ). Concernant le nombre de problèmes connus chez le parent, le même scenario se présente. Lorsque que l'on compte trois problèmes et plus connus chez le parent, se sont les parents autochtones qui sont surreprésentés $(25 \%$ vs $18 \%)$.

\begin{tabular}{|c|c|c|c|c|c|c|c|}
\hline $\begin{array}{l}\text { Tableau } 4 \\
\text { Facteurs associés aux signalements reter } \\
\text { hiérarchiques }\end{array}$ & impliquan & nfar & tone: & de $r$ & 100 & bar & \\
\hline Variables & $\begin{array}{l}\text { Rapport- } \\
\text { des cotes }\end{array}$ & & & Wald & prob & LR & prob \\
\hline Type de réception & & & & & & 7.920 & .0191 \\
\hline Urgence sociale /service régulier & 2.6525 & 1.2348 & 5.8275 & 6.2144 & .0127 & & \\
\hline Inconnu /service régulier & 7.3621 & 0.5433 & 99.7658 & 2.2535 & .1333 & & \\
\hline Source du signalement: mère & & & & & & 5.315 & .0211 \\
\hline Non/Oui & 3.0694 & 1.1582 & 8.1345 & & & & \\
\hline Retard de développement de l'enfant & & & & & & 4.638 & .0131 \\
\hline Non loui & 1.0028 & 1.0516 & 7.0667 & & & & \\
\hline Fratrie totale & & & & & & 8.501 & .0035 \\
\hline Pour chaque frère/sœur (0-5) & 1.3461 & 1.0944 & 1.6558 & & & & \\
\hline Problèmes d'abus d'alcool /drogue du parent & & & & & & 16.854 & .0000 \\
\hline Oui/non & 3.0753 & 1.7359 & 5.4483 & & & & \\
\hline Type d'occupation du logement & & & & & & 46.118 & .0000 \\
\hline Logement subventionné/log.non subventionné & 5.7954 & 2.47 & 13.60 & 16.30 & .0001 & & \\
\hline Propriétaire /logement non subventionné & 1.5746 & 0.68 & 3.62 & 1.14 & .2857 & & \\
\hline Autre/logement non subventionné & 12.31 & 3.99 & 38.05 & 19.02 & .0000 & & \\
\hline Inconnu / logement non subventionné & 8.00 & 2.52 & 25.47 & 12.42 & .0004 & & \\
\hline
\end{tabular}


Pour déterminer lesquelles de ces caractéristiques, différenciant les dossiers autochtones et non-autochtones, permettent de prédire qu'un signalement de protection de la jeunesse au Québec soit celui d'un enfant autochtone comparativement à un non-autochtone, nous avons effectué une analyse de régression logistique sur les deux catégories d'enfants signalés.

\section{Analyses multivariées}

Le tableau 4 présente les résultats du modèle de régression logistique des facteurs associés aux enfants d'origine autochtone signalés aux services de protection de la jeunesse du Québec. Le résultat du test d'ajustement de Hosmer et Lemeshow (1980) montre qu'il n'y a pas de différence significative entre les prédictions du modèle et les données observées (Goodness of Fit $=7.08 ; \mathrm{dl}=8 ; \mathrm{p}=.5278$ ), ce qui implique que le modèle obtenu reflète bien les données observées. En ordre décroissant d'importance, les facteurs suivants sont associés à une plus grande probabilité que le signalement implique un enfant autochtone: 1) le type d'occupation du logement, à savoir que les enfants autochtones vivent plus souvent dans un logement subventionné ou un autre type de logement; 2) plus de problèmes connus ou soupçonnés d'abus de substances chez le parent; 3) le nombre plus élevé d'enfants dans la famille; 4) le fait que le signalement soit reçu par les urgences sociales; 5) le fait que la source de signalement soit moins souvent la mère de l'enfant signalé; et 6) le fait que les enfants souffrent moins de retard de développement. Le type d'occupation du logement et les problèmes connus ou soupçonnés d'abus de substances chez le parent sont les variables caractérisant nettement le plus les signalements impliquant un enfant autochtone.

Le modèle de régression obtenu permet de prédire correctement $81 \%$ des signalements autochtones et nonautochtones. Le modèle prédit mieux les signalements non-autochtones $(86 \%)$ que ceux correspondant aux signalements autochtones (76\%).

Certaines variables significatives lors des analyses univariées n'ont pas été retenues par le modèle de régression. Ces variables sont: 1) la prise en charge antérieure (5ans); 2) la source de signalement provenant de la famille élargie; 3) les variables liées à la problématique fondée, telles que la présence de négligence et la présence de troubles de comportement;
4) le nombre d'enfants signalés dans la famille; 5) le niveau de coopération du parent; 6) les mauvais traitements vécus par les parents dans l'enfance; et 7) la présence de violence conjugale.

\section{Discussion \\ Forces et limites méthodologiques}

La présente étude est la première à brosser un portrait québécois des mauvais traitements envers les enfants autochtones signalés aux DPJ du Québec. Les analyses réalisées l'ont été à partir d'un échantillon représentatif des signalements impliquant des enfants autochtones pour la période et les régions couvertes par l'étude. Cette représentativité n'est toutefois pas parfaite car la courte période de collecte de données (automne 1998) pourrait avoir fait en sorte de surreprésenter ou de sous-représenter certains phénomènes plus saisonniers ${ }^{7}$. De plus, deux régions administratives ont été exclues de l'étude, soit le Nunavik et les Terres Cries de la Baie James, deux régions ayant une très forte population autochtone. Enfin, soulignons que l'ÉIQ n'a porté que sur les enfants signalés à la protection de la jeunesse et ne représente donc pas un portrait exact de la situation des mauvais traitements vécus par les enfants autochtones du Québec.

Une limite importante de ce type d'étude qui compare deux groupes ethniques est le biais lié aux définitions utilisées concernant les mauvais traitements envers les enfants. En effet, les définitions retenues sont davantage susceptibles de refléter la position des groupes ethniques majoritaires nord-américains. Il est donc possible que du point de vue des groupes autochtones, ces définitions ne correspondent pas exactement à celles retrouvées dans leur culture. Les plus grandes différences pourraient se retrouver au plan de la définition de la négligence et des troubles de comportement et, dans une moindre mesure cependant, au plan de la définition des abus physiques, des abus sexuels et surtout de l'inceste qui font généralement l'objet d'un consensus plus large à travers les cultures.

Par ailleurs, quelques forces intéressantes de cette étude sont à souligner, dont le fait d'avoir appareillé les deux groupes sur des variables liées au lieu de provenancedusignalementetàcertaines caractéristiques familiales, dont le revenu socioéconomique, permettant ainsi de mieux dégager des différences culturelles. Enfin, le fait d'avoir utilisé une approche d'analyses multivariées permettait de mieux isoler le rôle de chacune des variables à l'étude. 


\section{Pauvreté chez les familles autochtones sig- nalées en protection de la jeunesse au Québec}

Bien que les conditions de pauvreté des familles autochtones ne puissent expliquer les résultats issus de nos analyses comparatives, il est important de souligner que les familles autochtones de l'étude constituaient un échantillon représentatif des Autochtones signalés en protection de la jeunesse, alors que les non-Autochtones ne représentaient qu'un sous groupe de leur population dû à l'appariement des deux groupes. Le pourcentage de familles autochtones signalées à la protection de la jeunesse et affectées par un revenu annuel de moins de $15000 \$$ est de $39 \%$, en comparaison à $29 \%$ pour l'ensemble des familles québécoises signalées, démontrant que la pauvreté représente un enjeu majeur dans les situations autochtones signalées à la protection de la jeunesse et qu'elle représente une caractéristique importante de ces familles signalées. La mise en place de solutions au phénomène des mauvais traitements dans les communautés autochtones doit donc obligatoirement tenir en compte ce phénomène de pauvreté.

\section{Caractéristiques des signalements d'enfants autochtones}

Les résultats de l'étude nous démontrent qu'au Québec, les caractéristiques des signalements d'enfants autochtones diffèrent peu des signalements impliquant des enfants non-autochtones vivant dans des familles ayant un revenu familial équivalent. Seulement six des variables permettent de distinguer les signalements d'enfants autochtones. En effet, ces derniers se caractérisent par une proportion plus grande: 1) de signalements reçus par les urgences sociales; 2) de familles vivant dans un logement subventionné; 3) de familles constituées d'un plus grand nombre d'enfants; et 4) de parents présentant un problème d'abus de substances. De plus, les signalements d'enfants autochtones ont une proportion moins grande d'enfants présentant des problèmes de retard de développement et dont la source de signalement est la mère.

\section{Situation de crise}

Une première différence importante entre les deux groupes a trait au fait que les signalements d'enfants autochtones sont reçus deux fois plus souvent que les non-autochtones par les urgences sociales, c'est-à-dire en dehors des heures de services réguliers ( $8 \mathrm{~h}$ à $17 \mathrm{~h}$ ). Ces signalements pourraient se produire lors d'une crise familiale et provenir de l'entourage de la famille, dont les membres de la famille élargie et les membres de la communauté, deux sources qui ont signalé le quart des situations impliquant des enfants autochtones.

\section{Famille et logement}

Les familles autochtones signalées se caractérisent aussi par un nombre plus élevé d'enfants dans la famille et une proportion plus faible de familles vivant dans un logement non subventionné ou dont elles sont propriétaires. Cette surreprésentation est également présente concernant le nombre d'enfants par famille dans la population autochtone du Québec, comparativement à la population générale du Québec (Affaires Indiennes et du Nord Canadien, 2002). Ce plus grand nombre d'enfants est susceptible d'entraîner un stress additionnel pour les parents autochtones. Il est également attendu qu'il y ait moins de déménagements dans les familles autochtones compte tenu qu'une bonne proportion d'entre elles vivent dans une réserve et donc aurait un environnement social plus stable.

\section{L'abus de drogue ou d'alcool}

Les parents des enfants autochtones signalés sont plus nombreux à présenter un problème d'abus de substances, ce que rapportaient également Trocmé, Knoke et Blackstock (2004) dans l'étude canadienne. Le problème lié à l'abus d'alcool et de drogue apparaît particulièrement important dans les communautés autochtones, d'autant plus que la pauvreté s'avère un facteur de risque important de l'abus de substances (Commission royale sur les peuples autochtones, 1996; Santé Canada, 2006).

De plus, la consommation abusive d'alcool et de drogues chez les parents se révèle être également un facteur de risque connu de mauvais traitements envers les enfants (Bays, 1990; Famularo, Kinscherff \& Fenton, 1992; Kelleher, Chaffin, Hollenberg \& Fischer, 1994; Peterson, Gable \& Saldana, 1996; Trocmé, McPhee \& Tam, 1995). En effet, la toxicomanie, notamment, affecterait les capacités parentales en altérant les capacités de supervision parentale. (Dore, Doris \& Wright, 1995). D'ailleurs, dans l'ÉCI, les cas de violence psychologique et de négligence sont plus fréquents lorsque la consommation abusive d'alcool et de drogues des parents est rapportée. Plus spécifiquement, le risque de négligence était 1.5 fois plus élevé dans les familles où il y avait abus de substances chez les parents, que dans les familles où l'on ne rapportait pas de consommation abusive (Wekerle, Wall, Leung \& Trocmé, 2004). 
Bien que la présence de négligence ne soit pas identifiée dans les analyses multivariées comme une variable distinguant les deux groupes, la proportion d'enfants négligés dans le groupe d'enfants autochtones est plus élevée que celle du groupe d'enfants nonautochtones de notre étude. De plus, par rapport à l'échantillon total des enfants signalés de l'ÉIQ, où $35 \%$ de l'ensemble des enfants québécois signalés avaient été victimes de négligence, la proportion d'enfants autochtones impliqués dans une forme de négligence est nettement plus élevée, soit de $48 \%$. Cette prédominance de la négligence dans les familles autochtones avait également été constatée dans d'autres études et pourrait donc être associée aux problèmes d'abus de substances des parents. (Trocmé et al., 2004; McShane, 1988).

Dans le contexte des services de protection de l'enfance auprès de familles autochtones, il apparaît important de travailler auprès des parents présentant des problèmes de consommation abusive de drogue et d'alcool. Bien qu'il n'y ait pas encore de programmes de traitement de la toxicomanie ayant démontré clairement leur efficacité, Santé Canada a recensé un ensemble de programmes qui ont été implantés dans les communautés autochtones. Plusieurs de ces programmes ont été adaptés à la culture autochtone et semblent prometteurs.

\section{Retard de développement et troubles de comportements}

Les résultats montrent également que les enfants autochtones présentent dans l'ensemble moins de problèmes que les enfants non-autochtones. Notamment, la présence de retard de développement et de troubles de comportement chez l'enfant semble être davantage associée aux enfants non-autochtones.

Compte tenu de la présence plus importante de certaines caractéristiques familiales démontrées dans les analyses univariées, soit une plus grande proportion : 1) de parents ayant une consommation abusive de substances; 2) de parents ayant plus de trois problèmes; 3 ) de parents impliquées dans de la violence conjugale ou; 4) de parents ayant vécu des mauvais traitements durant leur enfance, il est étonnant de constater que les enfants autochtones présentent quant à eux moins de problèmes que les enfants des familles non-autochtones. Il est toutefois possible que les enfants autochtones puissent bénéficier du soutien de la famille élargie et ainsi être moins affectés par les problèmes de leurs parents. La stabilité plus grande (moins de déménagements chez les familles autochtones) pourrait expliquer la présence de ce soutien social que nous ne retrouverions pas chez les enfants non-autochtones. Ces résultats diffèrent de ceux de Trocmé et al. (2004), ces derniers ayant plutôt constaté que les enfants autochtones du Canada (excluant le Québec) présentaient davantage de problèmes que les enfants non-autochtones ${ }^{8}$. Les différences significatives se situaient au plan des handicaps à la naissance liés à l'abus de substances ainsi qu'au nombre de problèmes de comportement identifiés chez l'enfant.

\section{Similarités et différences notées entre l'ÉIQ et l'ÉCI}

Lesdonnéescomparatives pourl'ensembleduCanada (hormis le Québec) obtenues par Trocmé et al. (2004) concernant les mauvais traitements envers les enfants autochtones et non-autochtones signalés aux services de protection de l'enfance s'apparentent aux données comparatives obtenues au Québec, particulièrement concernant les caractéristiques familiales. En effet, la prédominance d'abus de substances, le plus grand nombre de problèmes et les mauvais traitements dans l'enfance sont davantage rapportés par les parents autochtones que les non-autochtones dans les deux études. Tel que présenté précédemment, certaines différences sont observées entre les données comparatives canadiennes et québécoises, principalement en ce qui a trait à la présence des troubles de comportements et des problèmes connus, ces derniers étant plus présents chez les enfants autochtones que les non-autochtones de l'ÉCI, alors qu'ils sont moins rapportés pour les enfants autochtones de l'EIQ en comparaison aux enfants non-autochtones.

Mais ces comparaisons entre les données comparatives canadiennes et québécoises doivent se faire avec prudence. En effet, il est difficile de comparer ces résultats pour différentes raisons, notamment, dû au fait : 1) que l'étude de Trocmé et al. (2004), contrairement à l'étude québécoise, n'a pas apparié l'échantillon de familles autochtones au niveau du revenu familial ; 2) que les deux échantillons canadien et québécois diffèrent, les familles autochtones de l'ÉCI semblant vivre davantage de difficultés psychosociales (plus de familles monoparentales, davantage de déménagements dans les 12 derniers mois, parents plus jeunes, consommation abusive de substances plus importante, plus d'activités criminelle 
et plus de problèmes de santé mentale chez les parents); 3) les analyses de Blackstock et al. (2004) incluent un troisième groupe ethnique, ce qui fait en sorte que nous ne pouvons pas préciser spécifiquement la différence entre Autochtones et non-Autochtones; et 4) que la définition de certaines variables, dont les troubles de comportements, diffèrent d'une étude à l'autre. Ainsi, certaines tendances peuvent être dégagées entre les deux études, sans toutefois pouvoir offrir de comparaisons parfaites.

\section{Enjeux pour la pratique}

Compte tenu que les parents autochtones ayant fait partie de notre étude étaient affectés par de multiples problèmes personnels (problèmes économiques, mauvais traitements vécus dans l'enfance, abus de l'alcool/drogue et violence conjugale) et vivaient dans certains contextes (nombreélevéd'enfants dans la familleetmonoparentalité) fréquemment associés aux risques de mauvais traitements (Schumacher et al., 2001), il est peu réaliste de penser que les situations de mauvais traitement et de troubles de comportement puissent cesser sans que des interventions intensives touchant à ces multiples aspects soient mises en place.

L'implantation de programmes de traitement dans les communautés autochtones doit toutefois tenir compte de la culture de ces communautés. Selon Morin et Joncas (2004), il semble n'y avoir aucune contreindication à l'utilisation, pour des enfants autochtones, d'un modèle de traitement ayant démontré sa valeur auprès d'enfants nord-américains. Cependant, Morin et Joncas (2004), tout comme plusieurs autres auteurs, s'entendent sur le fait que si les cibles et les techniques d'intervention sont transférables à la culture autochtone, certaines sources de tension sont susceptibles d'apparaître et de miner l'intervention si les intervenants non-autochtones ne modifient pas leur style interpersonnel pour tenir compte des valeurs autochtones (LaDue, 1994; Lafromboise, Trimble, \& Mohatt, 1990; Santé Canada, 2006; Solomon, Heisberger, \& Winer, 1981). Une approche privilégiant un degré de directivité plus grand que celui utilisé dans une thérapie conventionnelle avec les non-Autochtones semble être efficace dans le traitement auprès des Autochtones (Heilbron \& Guttman, 2000). D'autres auteurs ajoutent aussi que les thérapies familiales traditionnelles, centrées sur la famille nucléaire, ne sont pas appropriées et devraient être modifiées pour inclure la famille élargie (Carter \& Parker, 1991; Heilbron \& Guttman, 2000), notamment lorsque celle-ci montre une coopération adéquate pour la réussite du traitement. Un autre point à prendre en considération est le niveau d'acculturation des familles autochtones qui sont signalées, c'est-à-dire leur niveau de croyance aux valeurs traditionnelles de leur peuple (Connors \& Oates, 1997; Topper, 1992). Pour des Autochtones encore très attachés à la culture traditionnelle, différents éléments de cette culture devraient être considérés dans le traitement (Ashby, Gilchrist \& Miramontez, 1987; Heilbron \& Guttman, 2000). À cet égard, il a été suggéré de faire appel à l'intervention communautaire en ce qui concerne le soutien moral à fournir aux familles signalées (Heilbron \& Guttman, 2000). L'identification des alliances qui existent entre les familles et les clans de la communauté peut s'avérer fort utile, notamment pour réduire les sources de conflit que les familles sont susceptibles de rencontrer dans leur communauté. Sur le plan communautaire, le niveau d'attachement aux valeurs traditionnelles semble être encore plus important, puisque dans les communautés fortement traditionnelles, les intervenants non-autochtones pourraient avoir encore plus de difficulté à établir leur pratique (Darou, Kurtness \& Hum, 2000).

\section{Conclusion}

Bien que les résultats présentés puissent être utiles aux décideurs, aux intervenants ainsi qu'aux chercheurs, ils soulèvent également quelques questions supplémentaires qui pourraient être répondues dans les recherches futures. Il semble nécessaire d'approfondir la compréhension des phénomènes de maltraitance et des troubles de comportement chez les jeunes Autochtones, en examinant davantage les caractéristiques de ces problématiques prises isolément, soit les abus physiques, la négligence, l'abus sexuel et les mauvais traitements psychologiques. Il serait nécessaire également d'entreprendre une étude longitudinale auprès des enfants autochtones et nonautochtones pour qui les signalements sont retenus ou corroborés en services de protection, en vue d'approfondir les connaissances sur la trajectoire de ces enfants et de mieux répondre à leurs besoins et à ceux de leur famille.

Par ailleurs, il s'avère nécessaire également de mieux connaître les définitions et les formes de mauvais traitements reconnues par les membres de la 
communauté autochtone et de déterminer le degré de convergence pouvant exister avec celles attribuées par la DPJ. Les motifs et le contexte qui portent les membres de la famille élargie à signaler les enfants victimes de mauvais traitements devraient être également documentés.

\section{Notes Finales}

1. Le terme Autochtone est utilisé pour désigner les nations originaires du pays qu'elles habitent. Au Québec, les Amérindiens, les personnes d'ascendance amérindienne et les Inuits sont appelés Autochtones (Secrétariat aux affaires autochtones, 2001). Les Autochtones québécois se distinguent donc des Québécois de souche européenne, qui sont nés ici mais dont les ancêtres ont immigré à partir du 17 e siècle.

2. Les politiques d'assimilation des autochtones semblent s'être peu distinguées entre les diverses provinces canadiennes, notamment dans la mesure où les affaires autochtones relèvent depuis 1867 du gouvernement canadien.

3. "Pendant cette période on reconnaît généralement que le nombre total d'enfants autochtones placés sous les soins de personnes non-autochtones serait peut-être beaucoup plus élevé que les statistiques le révèlent si les enfants non inscrits et les enfants métis placés avaient été considérés comme des enfants autochtones dans ces statistiques. "(Bennett \& Blackstock, 2002).

4. Les Autochtones qui vivent dans les réserves et dans les trois territoires, soit environ $36 \%$ de la population autochtone, ne sont pas inclus dans les données. Comme le revenu est en général moins élevé pour la population autochtone qui vit dans les réserves comparativement à celle qui vit hors des réserves, l'inclusion de ces données ferait probablement augmenter l'incidence du faible revenu chez les enfants et les adultes autochtones.

5. L'Étude Canadienne d'Incidence $(E C I)$ a été réalisé auprès d'un échantillon représentatif de signalements fait aux services canadien de protection de l'enfance durant une période trois mois, 1er octobre au 31 décembre 1998 (Trocmé et al., 2001). L'échantillon considéré dans les analyses de Blackstock et al. (2004) exclut les signalements québécois.

6 . Pour de plus amples informations sur la méthodologie de l'étude, le lecteur peut consulter Tourigny et al. (2002).

7. Voir Tourigny et al. (2002) pour plus de détails concernant les forces et limites méthodologiques.

8. Dans l'ÉlQ, étant donné la spécificité de la LPJ, plusieurs des problèmes connus chez l'enfant auxquels réfèrent Trocmé et al. (2004) sont considérés dans la définition des troubles de comportements (et non dans la liste des problèmes chez l'enfant) afin de juger d'une situation de compromission de la sécurité et du développement de l'enfant, à l'instar des autres formes de mauvais traitements. Ces problèmes n'ont donc pas été comptabilisés sous la variable "nombre de problèmes de comportement" comme dans l'ÉCl. Malgré cette différence, les résultats de l'ÉlQ montrent moins de problèmes de comportements chez les enfants autochtones, contrairement à l'ÉCl qui en rapporte davantage chez les enfants autochtones que les non-Autochtones, confirmant une différence des résultats entre les deux études.

\section{Références}

Adrian, M., Payne, N., \& Williams, R.T. (1991). Estimating the effect of native Indian population on county alcohol consumption: The example of Ontario. Revue internationale sur la toxicomanie, 2,(5A- 6A), 731-65.

Affaires indiennes et du Nord canadien (2002). Données ministérielles de base - 2001. Ottawa: Ministère des Affaires indiennes et du Nord canadien.

Armitage, A. (1995). Comparing the policy of $A b$ original assimilation: Australia, Canada and New Zealand. Vancouver: University of British Columbia Press.

Ashby, M.R., Gilchrist, L.D., \& Miramontez, A. (1987). Group treatment for sexually abused American Indian Adolescents. Social Work with Groups, 10, 21-32.

Bays, J. (1990). Substance abuse and child abuse. Pedriatric Clinics of North America, 37, 881-904.

Bennett, M., \& Blackstock, C. (2002). A literature review and annotated bibliography focusing on aspects of Aboriginal child welfare in Canada. Ottawa: First Nations of Child and Family Caring Society of Canada.

Black, D.A., Heyman, R. E., \& Smith Slep,A. M.(2001). Risk factors for child physical abuse. Aggression and Violent Behavior, 6 (2-3), 203-229.

Blackstock, C., Trocmé, N., \& Bennett, M. (2004). Child maltreatment investigations among aboriginal and non-aboriginal families in Canada. Violence against Women, 10 (8), 901-916.

Bowlby, J. (2002). Niveau d'études post-secondaires au Canada et aux Etats-Unis dans les années 90. Ressources humaines et développement Canada. $\mathrm{N}^{\circ}$ de cat. MP32-30/02-2F-IN Site internet: https://:www.hrsdc.gc.ca/fr/sm/ps/rhdc/rpc/publications/recherche/2002-000014/page01.shtml.

Carter, I., \& Parker, L.J. (1991). Intrafamilial sexual abuse in American Indian families. In M.Q. Patton (Éd.), Family sexual abuse: Frontline research and evaluation (pp.106-117). Newbury Park, CA: Sage. 
Commission royale sur les peuples autochtones (1996). Vers un ressourcement / Gathering Strength (Rapport final), 166p.

Commission de la santé et des services sociaux des Premières Nations du Québec et du Labrador (2006). Enquête régionale longitudinale sur la santé des Premières Nations de la région du Québec 2002: Rapport des Premières Nations vivant hors communauté en milieu urbain. Siteinternet: http://www.cssspnql.com:8080/cssspnq1/ui/strategy/document/erlspn final hors communaute fr.pdf.

Connors, E.A., \& Oates J.M.L.B. (1997). The emergence of sexual abuse treatment models within first Nations communities. In D.A.Wolfe, R.J., McMahon, \& R.D. Peters (Éds.), Child Abuse: New Directions in prevention and treatment across the lifespan (p.223-247). Thousand Oaks, CA: Sage.

Conseil national du bien-être social. (2000). Profil de la pauvreté au Canada 1998, Ministère des ressources humaines et du développement social $d u$ Canada. Téléchargé à partir du site http://www. ncwcnbes.net/documents/researchpublications/ ResearchProjects/PovertyProfile/1998Report Fall2000/FRE/ReportFRE.htm.

Darou, W.G., Kurtness, J., \& Hum, A. (2000). The impact of conducting research with a First Nation. Canadian Journal of Counselling / Revue Canadienne de counseling, 34(1), 43-54.

Dore, M., Doris, J.M., \& Wright, P. (1995). Identifying substance abuse in maltreating families: A child welfare challenge. Child Abuse and Neglect, 19, 531- 543.

Dupuis, R. (2001). Quel Canada pour les Autochtones? Montréal: Boréal.

Famularo, R., Kinscherff, R., \& Fenton,T. (1992). Parental substance abuse and the nature of child maltreatment. Child Abuse and Neglect, 16, 475-483.

Farris-Manning, C., \& Zandstra, M, (2003). Children in care in Canada: A summary of current issues and trends with recommendations for future research. Child Welfare League of Canada, Ottawa. Site internet: http://www.cecw-cepb.ca/DocsEng/ ChildrenInCareMar2003Final.pdf.

Fournier, S., \& Crey, E. (1997). Stolen from Our Embrace: The Abduction of First Nations Children and Restoration of Aboriginal Communities. Vancouver: Douglas \& McIntyre.

Gelles, R. J., \& Hargreaves, E. F. (1990). Maternal employment and violence toward children. In M. A. Straus \& R. J. Gelles (Eds.), Physical Violence in American Families: Risk Factors and Adaptation to Violence in 8,145 Families (pp. 507-526). New Brunswick, NJ: Transaction Publishers.

Gouvernement du Canada (2005). Les jeunes canadiens: Qui sont-ils et que veulent-ils ? Service Canada. Site internet: http://www.youth. gc.ca/yoaux.jsp?\&lang $=$ fr \&geo $=402 \&$ flash $=1 \& \mathrm{t}$ a=1\&auxpageid $=846$.

Heilbron, C.L., \& Guttman, M.A. (2000). Traditional healing method with First Nations women in-group counseling. Canadian Journal of Counselling/ Revue Canadienne de counseling, 34(1), 3-13.

Hosmer, D. W., \& Lemeshow, S. (1980). Goodness of fit test for the multiple logistic regression model. Communications in Statistic, Theory and Method, A9, 1043-1069.

Hudson, P., \& McKenzie, B. (1985). Child Welfare and Native People: The Extension of Colonialism. The social worker, 49, (2):63-88.

Hudson, B. (1987). Manitoba's Indian Child Welfare Services. In J.S. Ismael \& R.J. Thomlison (Eds.), Dans The balance: perspectives on Social Services and Social Issues. Ottawa: Canadian Council on Social Development: 251-264.

Jiwani, Y. (2000). L'enquête sociale générale de 1999 sur la violence conjugale: une analyse. Center for Research on Violence against Women and Children. Vancouver, Canada.

Kelleher, K., Chaffin, M., Hollenberg, J., \& Fischer, E. (1994). Alcohol and drug disorders among physically abusive and neglectful parents in a community-based sample. American Journal of Public Health, 84(10), 1586- 1590.

Kimelman, E.C. (1985). No quiet Place. (Rapport final). Review Committee on Indian and Métis Adoptions and Placements, Winnipeg, Manitoba: Department of Community Services.

Kunz, J.L., Milan, A., \& Schetagne, S. (2001). Inégalité d'accès: profil des différences entre les groupes ethnoculturels canadiens dans le domaine de l'emploi, du revenu et de l'éducation, Ottawa, Canadian Council on Social Develop- 
ment (study prepared for the Canadian Race Relations Foundation).

LaDue, R.A. (1994). Coyote Returns: Twenty sweats does not an Indian expert make. Women and Therapy, 15, 93-111.

LaFromboise, T.D., Trimble, J.E., \& Mohatt, G.V.(1990). Counseling intervention and American Indian tradition: An integrative approach. Counseling Psychologist, 18, 628-654.

Leung, S., \& Carter, J. (1983). Cross-cultural study of child abuse among Chinese, Native Indians, and Anglo-Canadian children. Journal of Psychiatric Treatment \& Evaluation, 5, 37-47.

Lochhead, C., \& Shillington, R. (1996). Un profil statistique de la pauvreté urbaine. Ottawa: Conseil Canadien de Développement Social.

McSchane, D. (1988). An analysis of mental health research with American Indian youth. Journal of Adolescence, 11, 87-116.

Miller, J.R. (1996). Shingwauk's Vision: A History of Native Residential Schools. University of Toronto Press.

Ministère des Affaires Indiennes et du Nord Canada (2006). Analyse des conditions socioéconomiques, 1996 et 2001: indiens inscrits, indiens inscrits vivant dans une réserve et ensemble de la population canadienne. Catalogue no. R32-163/2001FPDF. Téléchargé à partir du site: www.ainc-inac. gc.ca

Ministère de la Santé et des Services Sociaux (1998). Manuel de référence sur la protection de la jeunesse. Québec: Gouvernement du Québec.

Morin, A., \& Joncas, J. (2004). L'intervention thérapeutique auprès d'enfants amérindiens victimes d'agression sexuelle. Psychologie canadienne, 45 (1), 43-55.

O'keefe, M. (1995). Predictors of child abuse in martially violent families. Journal of Interpersonal Violence, 10, 3-25.

Peterson, L., Gable, S., \& Saldana, L. (1996). Treatment of maternal addiction to prenvent child abuse and neglect. Addictive Behaviors, 21 (6), 789-801.

Réame, D.G., \& Maklem, P. (1994). Education for subordination redressing the adverse affects of residential schooling. Seven Generations: An Information Legacy of the Royal Commission on Aboriginal Peoples. CD Rom: Libraxas.

Santé Canada (2006). Stratégies relatives à l'évaluation des programmes de lutte contre l'abus des substances chez les autochtones: Examen de la question. Site internet: http://www.hcsc.gc.ca/fnih-spni/pubs/ads/literary examen review/index f.HTML.

Schetagne, S. (1999). La pauvreté dans les agglomérations urbaines du Québec. Conseil canadien de développement social.

Schumacher, J. A., Smith Slep, A. M., \& Heyman, R. E. (2001). Risk factors for child neglect. Aggression and Violent Behavior, 6, 231-254.

Secrétariat aux affaires autochtones du Québec (2001). Les Amérindiens et les Inuits du Québec: Onze nations contemporaines. Québec: Gouvernement du Québec.

Secrétariat aux affaires autochtones du Québec (2005). Statistiques des populations autochtones du Québec. Québec: Gourvernement du Québec.

Sedlak, A.J. (1991). National incidence and prevalence of child abuse and neglect: 1988. Rockville, MD: Westat.

Sedlak, A.J. (1997). Risk factors for the occurrence of child abuse and neglect. Journal of Aggression, Maltreatment, \& Trauma, 1, 149-187.

Sedlak, A.J., \& Broadhurst, D.D. (1996). Third National Incidence Study of Child Abuse and Neglect. Washington, D.C.: National Center on Child Abuse and Neglect.

Solomon, G., Heisberger, J., \& Winer, J.L. (1981). Confidentiality issues in rural community mental health. Journal of Rural Community Psychology, 2, 17-31.

Statistique Canada (2003). Enquête auprès des peuples autochtones de 2001- Premiers résultats: Bien-être de la population autochtone vivant hors-réserve. Catalogue no. 89-589-XIF

Statistique Canada (2003). Recensement de 2001, série " analyses ». Peuples autochtones $d u$ Canada: un profil démographique. Catalogue no. 96F0030XIF2001007. 
Tiechroeb, R. (1997). Flowers on My Grave : How an Ojibway Boy's Death Helped Break the Silence on Child Abuse. Toronto: Harper Collins Publishers Ltd.

Topper, M.D. (1992). Multidimensional therapy: a case study of Navajo adolescent with multiple problems. In L.A. Vargas \& J.D. Koss-Chioino (Éds.), Working with culture: Psychoterapeutic interventions with ethnic minority children and adolescents (pp .225-245). San Francisco, CA : Jossey-Bass.

Tourigny, M., Mayer, M., Wright, J., Lavergne, C., Trocmé, N., Hélie, S., Bouchard, C., Chamberland, C., Cloutier, R., Jacob, M., Boucher, J., \& Larrivée, M.-C. (2002). Étude sur l'incidence et les caractéristiques des situations d'abus, denégligence, d'abandon et de troubles de comportement sérieux signalées à la direction de la protection de lajeunesse au Québec (ÉIQ). Montréal: Centre de Liaison sur l'Intervention et la Prévention Psychosociales.

Trocmé, N., Knoke, D., \& Blackstock, C. (2004). Pathways to the Overrepresentation of Aboriginal Children in Canada's Child Welfare System. Social Service Review, 78 (4), 577-600.

Trocmé, N., MacLaurin, B., Fallon, B., Daciuk, J., Billingsley, D., Tourigny, M., Mayer, M., Wright, J., Barter, K., Burford, G., Hornick, J., Sullivan, R., \& McKenzie, B. (2001). Étude canadienne sur l'incidence des signalements de cas de violence et de négligence envers les enfants: rapport final. Ottawa, Ontario: Ministère de Travaux publics et Services gouvernementaux Canada.

Trocmé, N., McPhee, D., \& Kwam Tam, K. (1995). Child abuse and neglect in Ontario: Incidence and characteristics. Child Welfare, 74 (3), 563- 586.

Trocmé, N., McPhee, D., Tam, K. K., \& Hay, T. (1994). Ontario incidence study of reported child abuse and neglect. Toronto, ON: Institute for the Prevention of Child Abuse.

Trudeau, A., \& Pellan, R. (1998). Définitions des termes et concepts utilisés dans la ventilation de la table des valeurs des articles 38 et 38,1 de la Loi sur la protection de la jeunesse. Montréal: Système clientèle jeunesse.

Vézina, A., \& Bradet, D. (1990). Inventaire concernant le bien-être de l'enfant en relation avec l'exercice des responsabilités parentales. Qué- bec: MSSS et Centre de recherche sur les services communautaires.

Wekerle, C., Wall, A-M., Leung, E., \& Trocmé, N. (2004). Cumulative stress and substantiated maltreatment: The importance of caregiver vulnerability and adult partner violence. Journal of Child Abuse \& Neglect. 31(4), 427-443

Zuravin, S. J. (1987). Unplanned pregnancies, family planning problems, and child maltreatment. Family Relations, 36(2), 135-139. 